International Journal of Wireless \& Mobile Networks (IJWMN) Vol. 4, No. 4, August 2012

\title{
VERTICAL HANDOFF IN WLAN-WIMAX-LTE HETEROGENEOUS NETWORKS THROUGH GATEWAY RELOCATION
}

\author{
L. Nithyanandan ${ }^{1}$ and I. Parthiban ${ }^{2}$ \\ ${ }^{1,2}$ Department of Electronics and Communication Engineering, \\ Pondicherry Engineering College, Puducherry, India \\ nithi@pec.edu \\ iparthi@pec.edu
}

\begin{abstract}
In wireless communication new technologies emerges regularly with faster data rates and larger coverage area. Therefore the forthcoming challenge is to make the best possible use of the available heterogeneous network. For connecting mobile between heterogeneous networks vertical handoff is mandatory. IP Multimedia Subsystem is an emerging architecture for interworking of heterogeneous networks. In this paper we used WLAN/WiMAX/LTE heterogeneous networks. Coupling architectures such as tight coupling and loose coupling are considered. In order to reduce the latency two mechanisms such as neighbour bandwidth reservation and gateway relocation are employed. The parameters such as vertical handoff delay, Mobile scanning interval activity, neighbouring advertisement received are obtained. From the simulation it is inferred that tight coupling with gateway relocation is performing better from handover point of view.
\end{abstract}

\section{KEYWORDS}

Vertical Handoff, IMS, WiMAX, WLAN \& LTE.

\section{INTRODUCTION}

Wireless technologies such as LTE, WLAN, WiMAX, etc were developed with different standards and these technologies offer variety of services, different data rates and diverse area of coverage. One of the forthcoming challenge in network management is to connect between end to end heterogeneous wireless technologies. To provide such end to end connection between heterogeneous networks we need to perform vertical handoff. If the serving and target base station during handoff are of different wireless technologies then such a handoff is called as vertical handoff. The term interworking is used to express interactions between heterogeneous networks with the aim of providing an end-to-end communication.

The IEEE 802.11 standard provides less cost and wireless LAN service effectively. The deployment of high speed network (11Mbps in $802.11 \mathrm{~b}$ and $54 \mathrm{Mbps}$ in $802.11 \mathrm{a} / \mathrm{g}$ ) can be established by unlicensed spectrum $(2.4 \mathrm{GHz}$ in $802.11 \mathrm{~b} / \mathrm{g}$ and $5 \mathrm{GHz}$ in $802.11 \mathrm{a})$. The IEEE $802.11 \mathrm{~b}$ standard for the WLAN can offer coverage of $100 \mathrm{~m}$. Access Point is an entity which function similar to base station is used in an Infrastructure type WLAN network. Another emerging wireless technology, WiMAX is based on the IEEE 802.16e standard. WiMAX base stations can offer greater coverage area about $8 \mathrm{~km}$ with data rate of 70Mbps. The LTE standard was developed by $3 \mathrm{GPP}$ with data rate of $150 \mathrm{Mbps}$. LTE can support inter system handover between LTE and 3G latency systems such as UMTS, GSM, etc. 
International Journal of Wireless \& Mobile Networks (IJWMN) Vol. 4, No. 4, August 2012

The rest of the paper is organized as follows. Section 2 gives the previous works. The interworking protocol used i.e. IMS is explained in section 3, It also deals with the methods employed to reduce handoff delay. Section 4 deals with the simulation and the proposed architecture. Section 5 explains the performance analysis and section 6 concludes with the future work.

\section{LITERATURE SURVEY}

W. Lee, et al., [1] has proposed radio signal strength based movement aware vertical handover algorithm between IEEE 802.11 WLAN and IEEE 802.16e Mobile WiMAX. Later vertical handover technology for mobile WiMAX systems and Long Term Evolution [2] was proposed to provide optimized handover using the data forwarding function in the network architecture. Further Vertical Handoff Decision algorithm [3] was proposed with load balancing mechanism over the base stations to optimize cost function involving battery life time of the mobile nodes (MN). Subsequently it is proved that Session Initiation Protocol (SIP) based IP Multimedia Subsystem registration (IMS) can provide session mobility with optimal service [5]. Optimal vertical handover (VHO) in a vehicular network was proposed recently in 2011 to optimize the cost of transmission [4]. Virtual partitioning with pre-emption technique in cellular/ IEEE 802.16e interworking [6] suggest admission control algorithms through IMS for connection requests considering the class of service (i.e., RT or NRT). In 2012 gateway relocation [7] mechanism is employed in homogeneous WiMAX networks to minimize handover delay and packet loss.

\section{IP MUlTiMEdiA SUBSYSTEM}

This session briefly explains about the interworking framework used i.e. IP multimedia subsystems and the various methodologies used to reduce handover delay.

\subsection{IP Multimedia Subsystem}

IP Multimedia Subsystem (IMS) is an emerging architectural framework based on SIP protocol, for offering multimedia services and VoIP services. IMS has been standardized by the 3GPP in order to support functionalities in both cellular and fixed technologies. IMS network consists of application layer to provide the end user with service controls and required services, control layer is responsible for delivery of control signals and connectivity or transport layer for transporting different types of information such as voice, data and multimedia streams. The main elements of IMS architecture shown in Figure 1 are as follows:

\subsubsection{The Call Session Control Functions}

SIP signaling in the IMS is processed by a SIP server called Call Session Control Function (CSCF) [9]. The three types of CSCFs are; Proxy Call Session Control Function (P-CSCF), Interrogating Call Session Control Function (I-CSCF) and Serving Call Session Control Function (S-CSCF).

\subsubsection{Home Subscriber Server}

Home Subscriber Server (HSS) is main data storage for all subscribers information such as user identities, registration information and service-triggering. It performs authentication and authorization of the user and provides information about the users physical location.

\subsubsection{Application servers}

It executes services and interfaces with the S-CSCF using Session Initiation Protocol. 
International Journal of Wireless \& Mobile Networks (IJWMN) Vol. 4, No. 4, August 2012

\subsubsection{Media Resource Function}

Media Resource Function (MRF) performs multiparty call, multimedia conferencing, tones and announcement functionalities. The MRF communicates with the S-CSCF for service validation of multiparty or multimedia sessions.

\subsubsection{Media Resource Function Controller}

Media Resource Function Controller (MRFC) performs processing of media streams through the corresponding Media Resource Function Processor (MRFP).

\subsubsection{Media Gateway Control Function}

Media Gateway Control Function (MGCF) communicates with the CSCF through SIP to control media channels for connection in a Media Gateway Function (MGW).

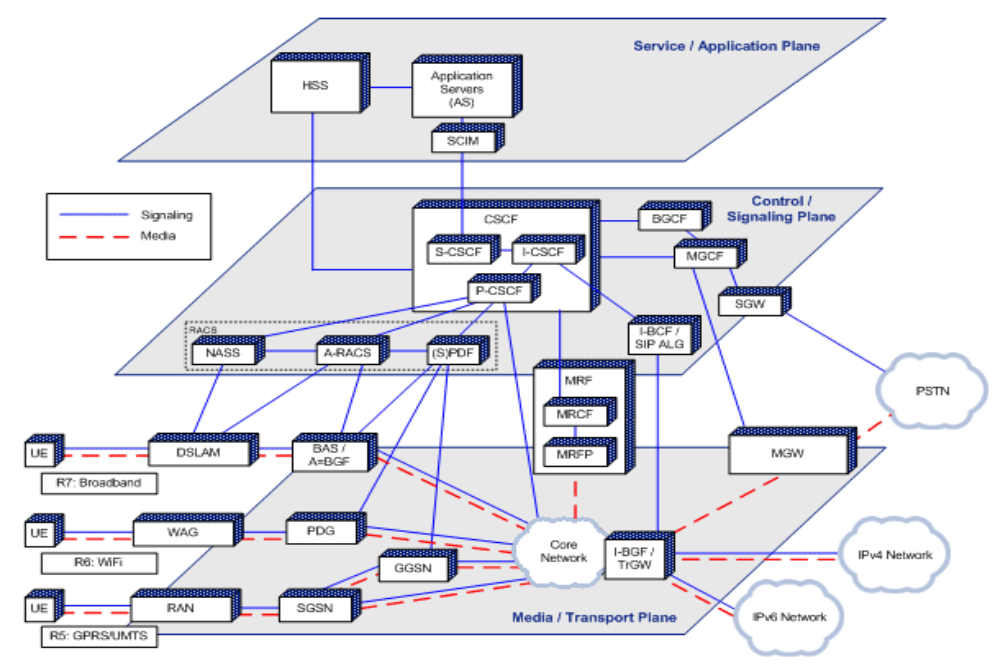

Figure 1. IMS architecture.

\subsection{Neighbour Bandwidth Reservation}

During handover the mobile node will switch from one base station to another base station. The handover is completed only when the new base station allocates bandwidth for the mobile node. The time taken to allocate bandwidth for the mobile node will increase the handover delay. Usually in voice communication the delay between the end users should be less than the voice inactivity time $(0.02 \mathrm{~s})$ such that the user will not identify that delay has taken place between the conversation. To avoid such handover delay the neighbour base stations are made such that the bandwidth is reserved for handover users hence the time taken to allocate resource for handover mobile node is reduced and therefore handover delay is reduced.

\subsection{Gateway Relocation Mechanism}

The architecture is depicted in Figure 2. The Access Service Network Gateway (ASN GW) provides wireless radio access for subscribers. It consists of corresponding gateway and base stations (BSs). Each gateway is connected to Session Initiation Protocol Gateway (SIP GW) which provides connectivity services. To support IP mobility IMS is adopted. Using gateway relocation when the MS roams from ASN GW to WAG, the ASN GW A will tunnel traffic to Wireless Access Gateway (WAG). The MS is still served by two GWs (ASN GW and WAG) as 
International Journal of Wireless \& Mobile Networks (IJWMN) Vol. 4, No. 4, August 2012

shown in Figure 2. Two-tiered mobility management defined in WiMAX potentially can minimize handover delay and packet loss. To establish the tunnel, Multi Protocol Label Switching (MPLS) is used as the private tunneling link.

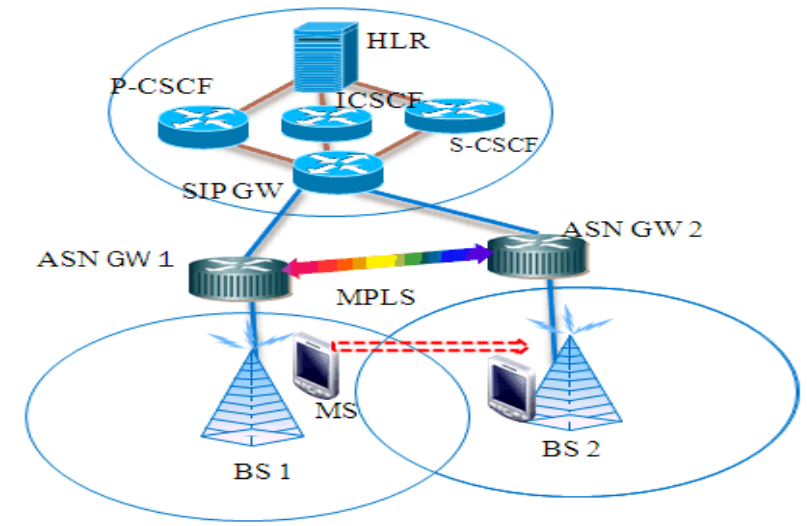

Figure 2. Gateway relocation mechanism.

\section{Proposed Architectures}

All current architectures in proposed wireless heterogeneous networks were deployed in an assumption that the network layer protocol used is IP. Applications and Transport layers are also IP layer compatible. The intention of WiMAX-WLAN-LTE interworking is to extend the services capabilities of WiMAX network also to WLAN and LTE environment and vice versa.

\subsection{Vertical Handoff Algorithm Based on Gateway Relocation}

Although vertical handoff is relatively new several studies still can be seen in literature [8]. In this paper we have used signal strength based handoff initiation as mobility is considered. In gateway relocation mechanism the gateways of the serving base station (LTE eNodeB) and target base station (here WLAN AP) are connected through Multi Protocol Label Switching tunnel. The various steps involved in the vertical handoff algorithm shown in Figure 3 are discussed below:

i) Consider the mobile node (MN) moves from the serving base station (here LTE eNodeB), then the radio signal strength of the base station will reduce beyond the threshold and data flow will cut off.

ii) In order to continue the ongoing data session the mobile node will initiate handoff request to the neighbouring base station (here WLAN AP). The WLAN GW will forward the request in the form of SIP REGISTER message to the P-CSCF. The $\mathrm{P}-\mathrm{CSCF}$ will forward the MN address to the I-CSCF.

iii) I-CSCF will send Diameter User Authentication Request (UAR) to the Home Subscriber Station (HSS) for authentication and also for determination of S-CSCF.

iv) The Home Subscriber Station (HSS) will authenticate the user by sending Diameter User Authentication Accept (UAA) to the I-CSCF.

v) I-CSCF will forward the SIP REGISTER request to the S-CSCF.

vi) After the request of I-CSCF the S-CSCF will request using Diameter Server Assignment Request (SAR) to the HSS.

vii) HSS will respond through Diameter Server Assignment Answer (SAA). 


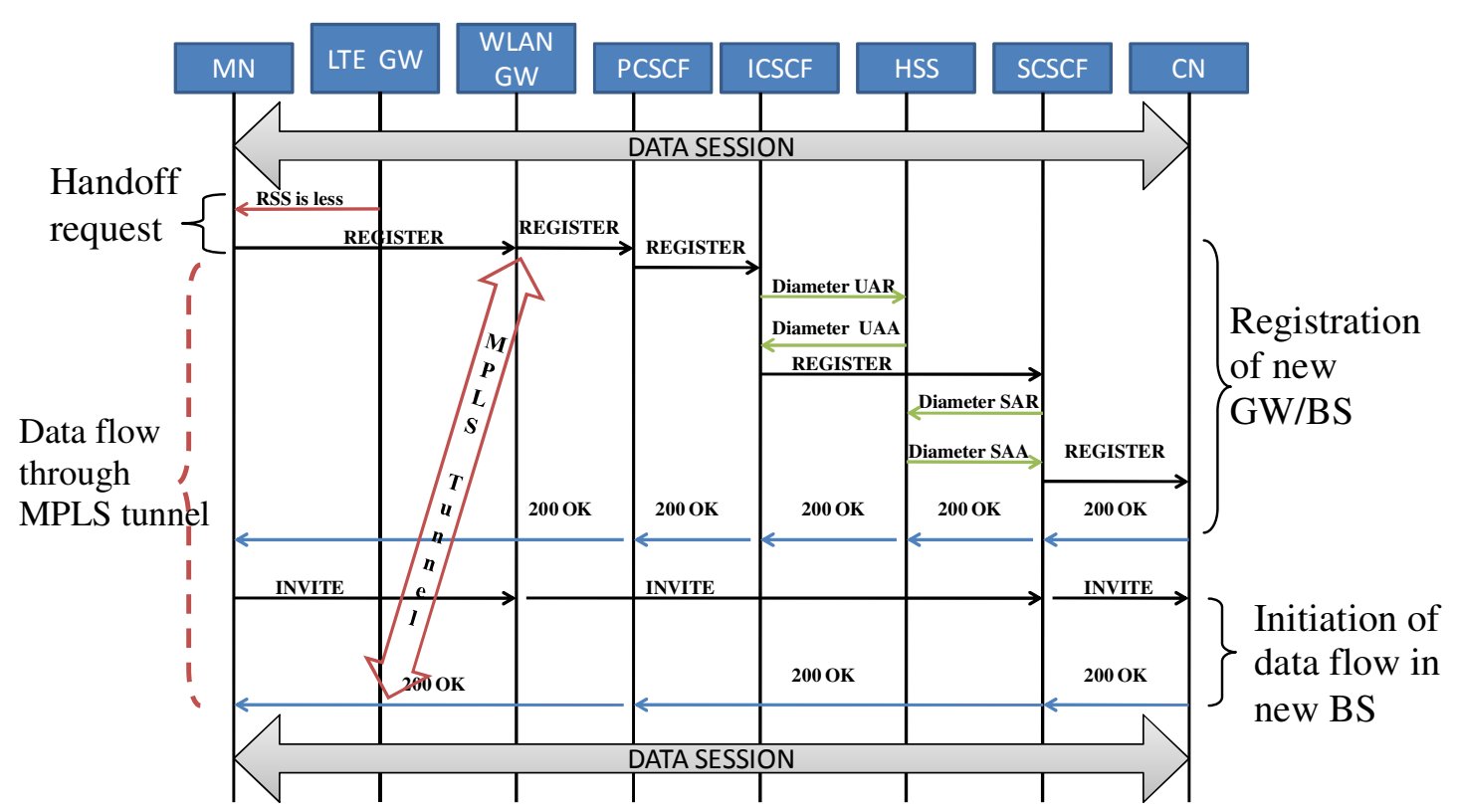

Figure 3. Vertical Handoff Algorithm based on gateway relocation.

viii) The Correspondence Node (Callee) will be notified by SIP REGISTER from the S-CSCF.

ix) Now all the nodes will be acknowledge by 200 OK to notify that the user is registered successfully to the new base station.

$\mathrm{x}$ ) The mobile node will initiate ongoing transfer by sending SIP INVITE message to the WLAN AP, which is again forwarded to the S-CSCF and then to the CN. The CN will reply through $200 \mathrm{OK}$ to continue the ongoing data session.

xi) By the use of gateway relocation methodology, during the handoff registration process also (interval between step ii to step $\mathrm{x}$ ) dataflow will continue, due to the reason that MPLS tunnel will assist data flow between LTE GW and WLAN GW. The data flow will not be disturbed until a new base station is registered.

xii) Now the data session will continue through the new base station.

Now for analysing the methodology four different scenarios have been simulated. The architectures employed includes: tight coupling, loose coupling, bandwidth reservation and gateway relocation.

\subsection{Tight Coupling}

In tightly coupled interworking the WLAN network is connected to the WiMAX network directly and also with LTE networks and appears to be one of the core networks. All the WLAN traffic is injected into the core network directly. This type of connection tends to be quicker in data transfer hence the delay will be less. The simulated architecture of WiMAX-WLAN-LTE tight coupled interworking is shown in Figure 4. 


\subsection{Loose Coupling}

The simulated architecture of WiMAX-WLAN loose coupled interworking is shown in Figure 5. In loose coupled interworking the WLAN network connected to the WiMAX and LTE network indirectly and appears to bypass through intermediate. All the WLAN traffic is injected into the intermediate network directly and from the intermediate network the traffic is sent to the core network. This approach gives independent deployment of WLAN, WiMAX and LTE networks.

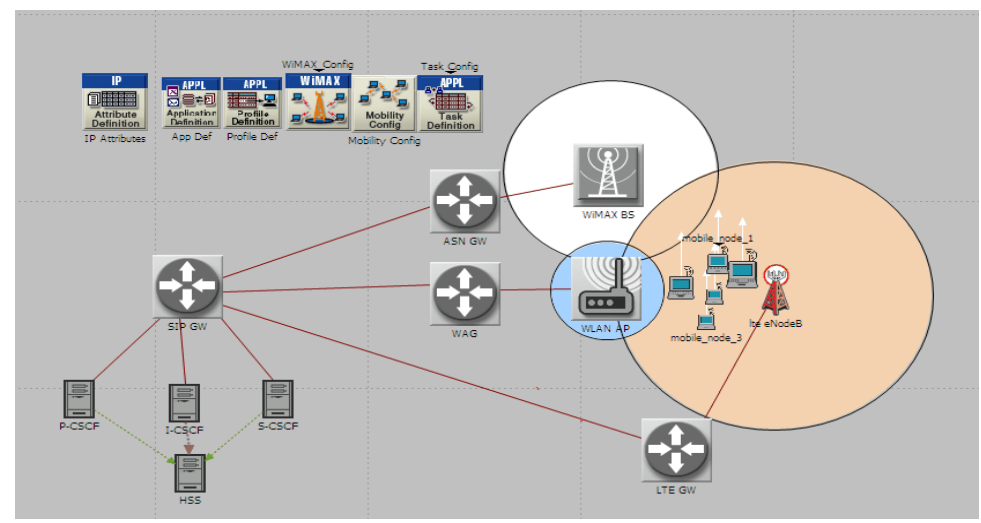

Figure 4. WiMAX-WLAN-LTE tight coupled interworking.

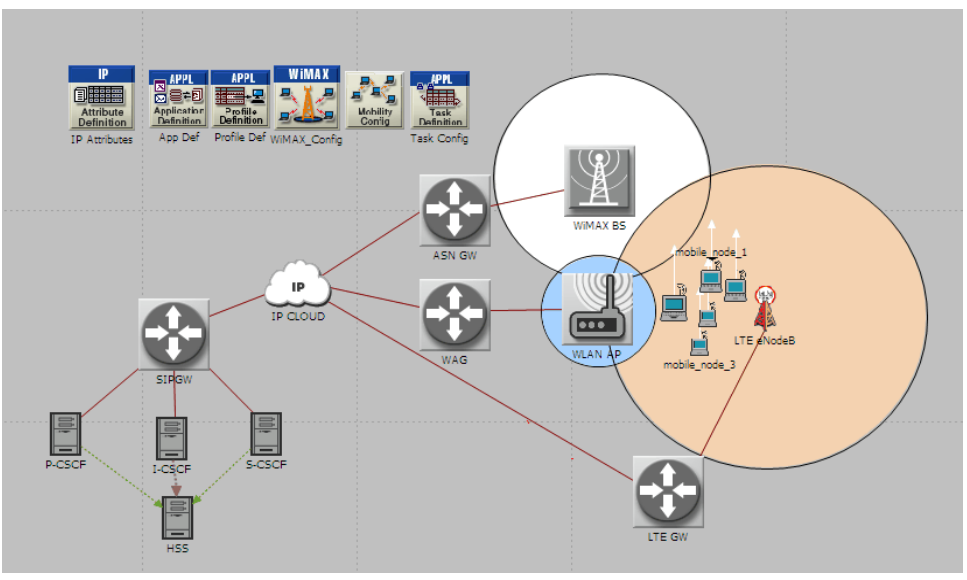

Figure 5. WiMAX-WLAN-LTE loose coupled interworking.

\subsection{Neighbour Reservation}

In tightly coupled interworking the WLAN and LTE networks are connected to the WiMAX network directly. In this network the bandwidth of all the network resource are reserved about $20 \%$ for the handover users so the time taken to allocate bandwidth for the mobile node will reduce. 


\subsection{Gateway Relocation}

The architecture is depicted in Figure 6. The heterogeneous wireless radio access technology consists of different gateway and corresponding base stations. Each gateway is connected to Session Initiation Protocol Gateway (SIP GW) which provides connectivity services. The different gateways are tunneled through Multi Protocol Label Switching. MPLS can encapsulate packets of various network protocols.

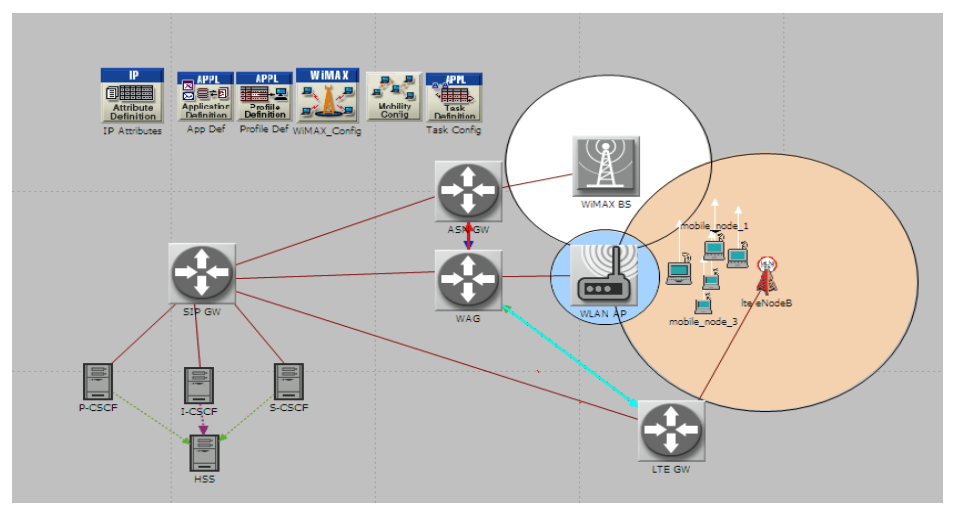

Figure 6. WiMAX-WLAN-LTE network with gateway relocation.

\section{Performance Analysis}

The software used to simulate the WiMAX-WLAN-LTE vertical handoff is OPNET Modeler. Using OPNET Modeler various simulation architectures such as tight coupling, loose coupling, tight coupling with neighbour bandwidth reservation and tight coupling with gateway relocation were simulated. The bandwidth reserved is $20 \%$ and for gateway relocation the link used for virtual tunneling is MPLS. The services that have been considered are voice service $(5.3 \mathrm{kbps})$, video conferencing $(1.93 \mathrm{Mbps})$, ftp service $(390 \mathrm{kbps})$, printing service $(70 \mathrm{Mb})$ and remote login service (125 bytes/sec).

\subsection{Transmitted Power}

Figure 7 shows the power required to transmit signal and it will be usually high for mobile which receives signal continuously and hence it is high for tight coupling and tight coupling with gateway relocation. 
International Journal of Wireless \& Mobile Networks (IJWMN) Vol. 4, No. 4, August 2012

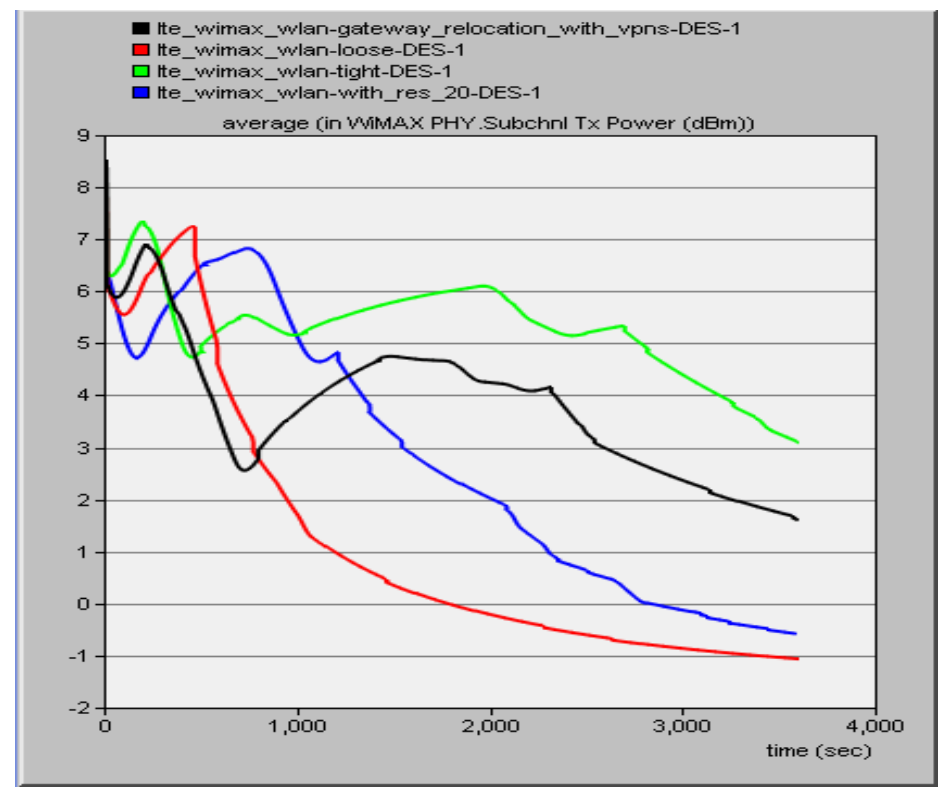

Figure 7. Transmitted power required.

\subsection{Serving Base Station Identity}

Figure 8 shows the serving base station identity no. during the simulation for all the above considered architectures. The result shows the base station id at which the mobile is being served ( 0 - no connection, 1- served by WiMAX base station, 2- served by WLAN access point and 3- served by LTE eNodeB).

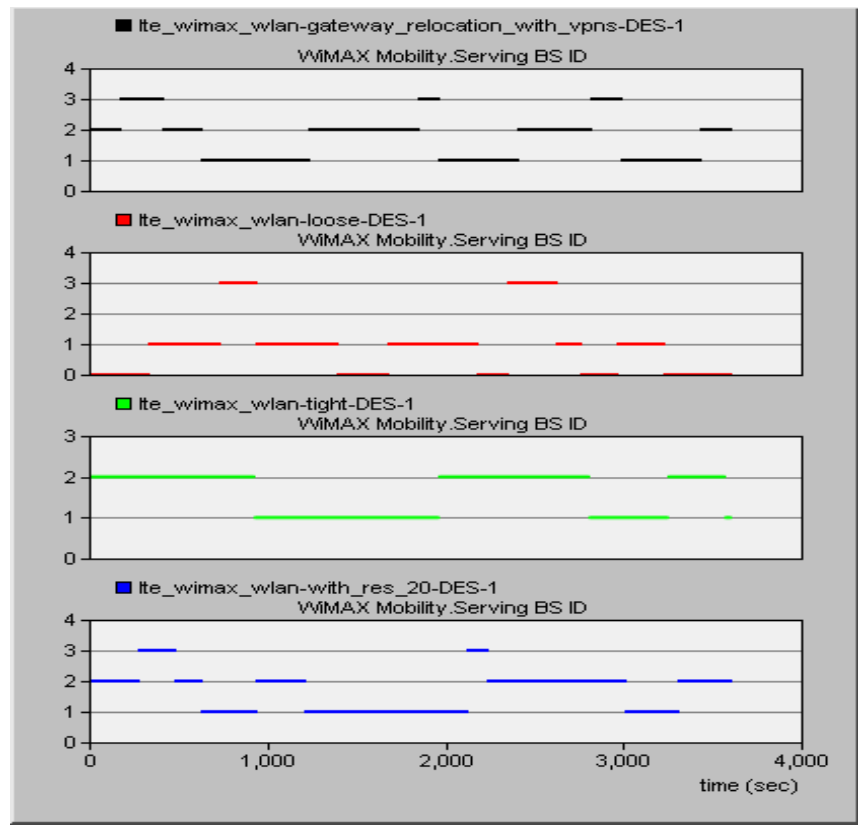

Figure 8. Serving base station identity. 


\subsection{Handover Delay}

Vertical handover delay of the interworking architecture for different services is simulated. Figure 9 and Figure 10 represents the handover delay for voice and video services respectively. It is found that with neighbour reservation handover delay is lesser since the time taken to allocate bandwidth for the mobile node is null.

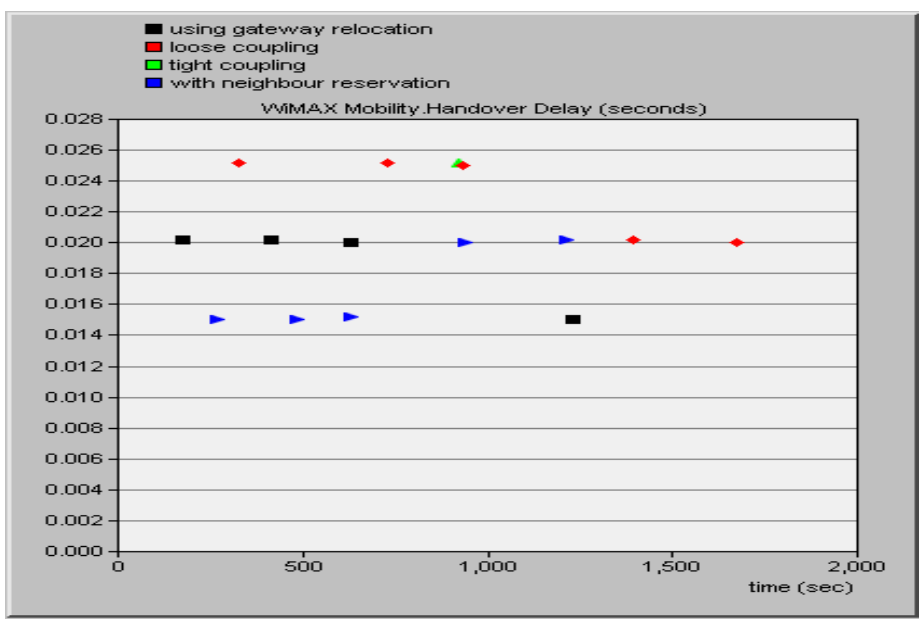

Figure 9. Handover delay for voice service.

It is also inferred that handover delay for gateway relocation is low. The mobile node moving from one network to another network need not wait until registration is completed in the new network. The new networks gateway will tunnel traffic to the already serving gateway through the tunnel established. The ongoing traffic is routed via this tunnel reducing the handover delay and packet loss.

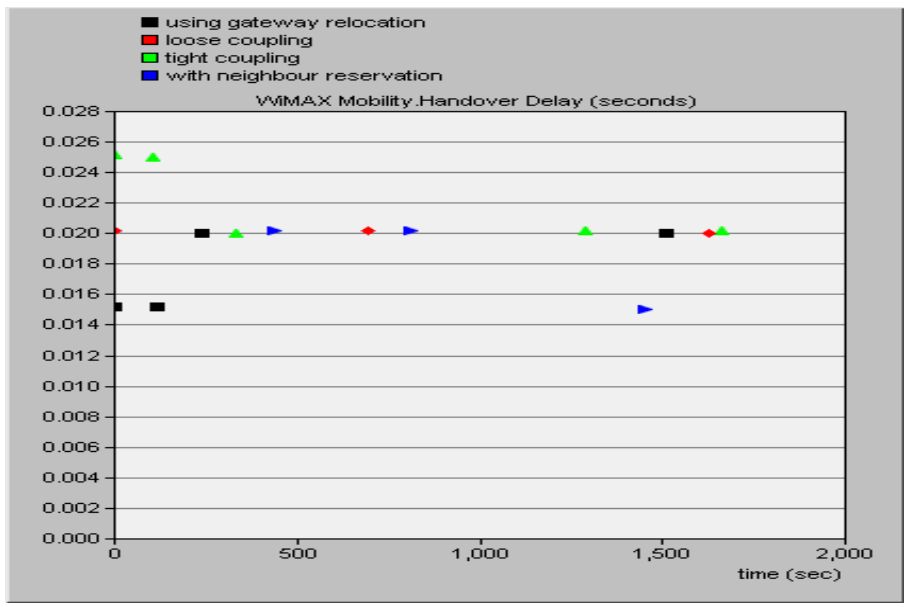

Figure 10. Handover delay for video service. 
International Journal of Wireless \& Mobile Networks (IJWMN) Vol. 4, No. 4, August 2012

\subsection{Signal to Noise Ratio}

Figure 11 shows the signal to noise ratio of the signal received by all the four architectures. It was found that the signal strength of gateway relocation is better since the no. of packets dropped is less.

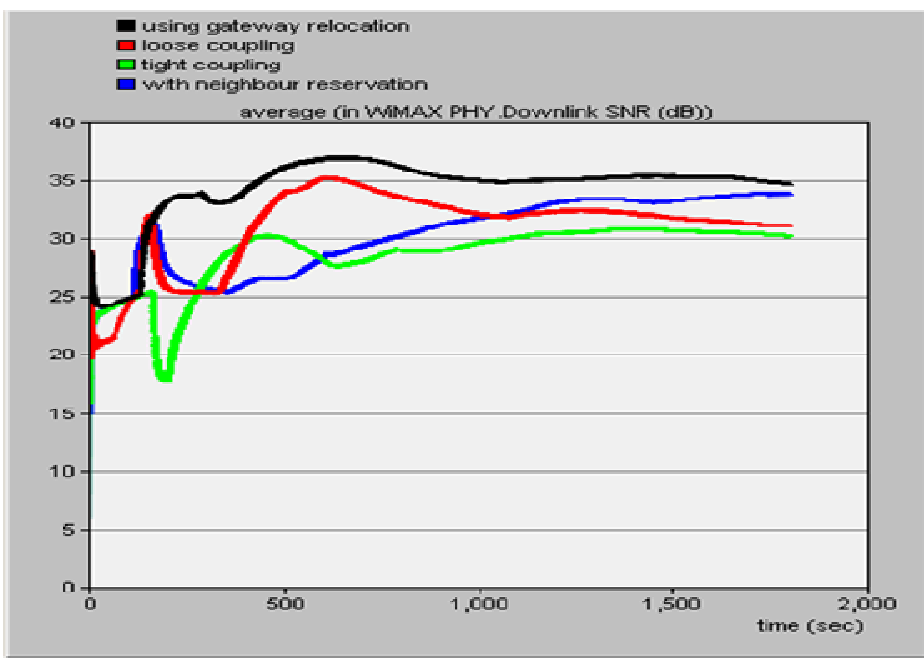

Figure 11. Signal to noise ratio at the base station.

\subsection{Packets Dropped}

Figures 12 and 13 show the packet dropped for both downlink and uplink respectively by the mobile node during vertical handoff. Its value is less for gateway relocation architecture since secondary path is already available for the ongoing traffic to flow before handover registration is completed at the new network.

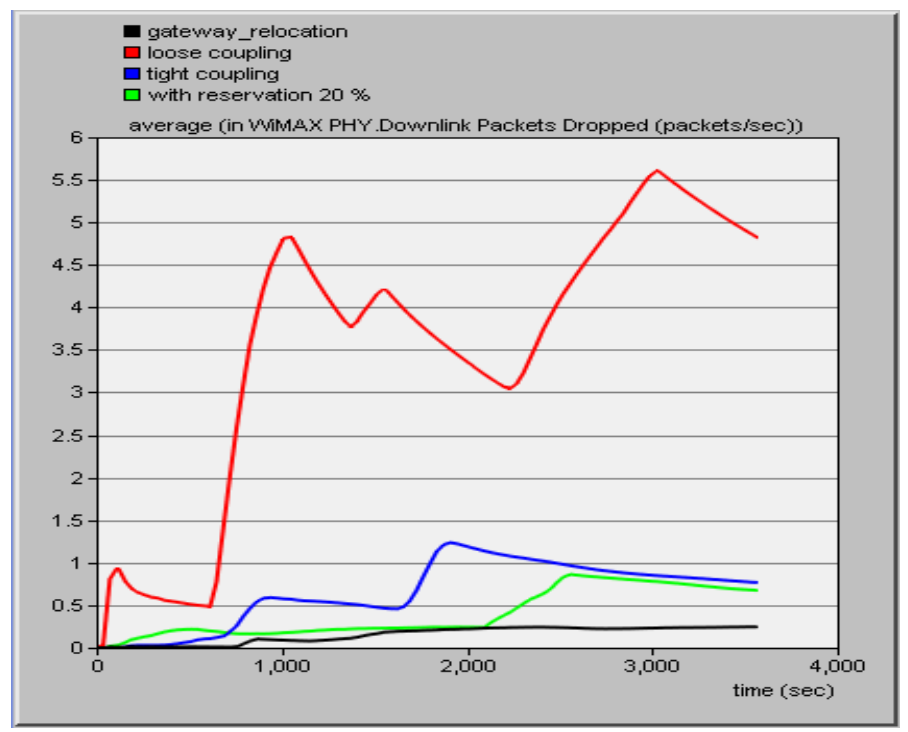

Figure 12. No. of packets dropped in downlink. 
International Journal of Wireless \& Mobile Networks (IJWMN) Vol. 4, No. 4, August 2012

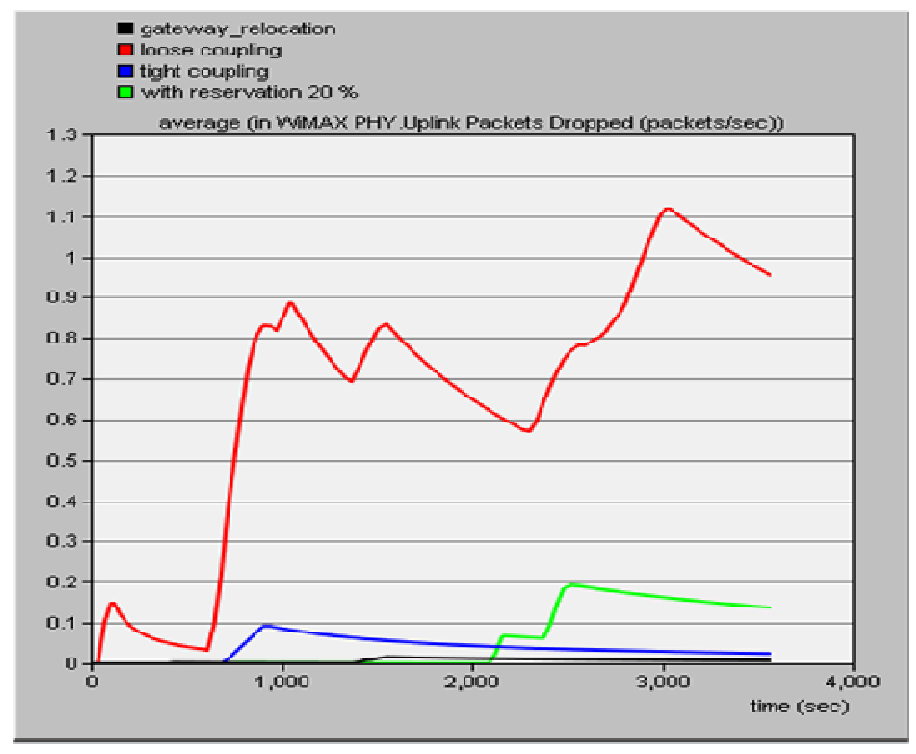

Figure 13. No. of packets dropped in uplink.

\subsection{Handover Delay vs Velocity}

The velocity of the mobile node is varied from $0 \mathrm{~m} / \mathrm{s}$ to $25 \mathrm{~m} / \mathrm{s}$ (up to $95 \mathrm{~km} / \mathrm{hr}$ ) in all the architectures and the average handover delay is obtained as in Figure 14. It is found that the handover delay of the neighbour reservation method and gateway relocation mechanism provides value well below the voice inactivity time $(0.02 \mathrm{~s})$ of normal voice communication.

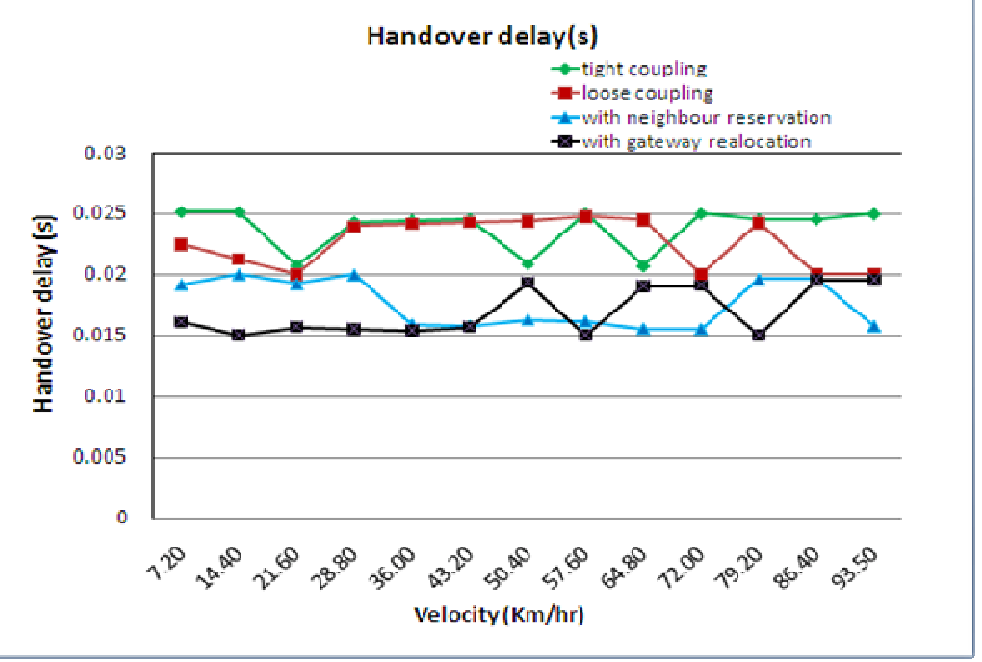

Figure 14. Handover delay with varying speed. 
International Journal of Wireless \& Mobile Networks (IJWMN) Vol. 4, No. 4, August 2012

\section{CONCLUSION}

Heterogeneous mobile networks such as WLAN, LTE and WiMAX require efficient handoff mechanisms to guarantee seamless connectivity. In this work four different types of interworking architectures were designed between WLAN, LTE and WiMAX networks namely: tightly coupled integration, loosely coupled integration, tight coupling with neighbour reservation and with gateway relocation. Consideration of vertical handover is made by locating the mobile node in a region where WLAN, LTE and WiMAX coverage coexist. It was found that tight coupling with neighbour reservation and with gateway relocation provides better handover performance. The network simulation also shows that interworking architecture with gateway relocation outperforms the other coupling methodologies due to the reason that a secondary path is established prior to handover and it results in less handover delay, lesser packets dropped and high signal to noise ratio. It was found that handover delay is lesser than the voice inactivity time with neighbour reservation and with gateway relocation and hence we can assure that seamless connectivity can be achieved.

\section{REFERENCES}

[1] W. Lee, E. Kim, J. Kim, I. Lee and C. Lee, "Movement-Aware Vertical Handoff of WLAN and Mobile WiMAX for Seamless Ubiquitous Access," IEEE Transactions on Consumer Electronics, vol. 53, no. 4, pp. 1268-1275, Nov. 2007.

[2] Kumudu S. Munasinghe and Abbas Jamalipour, "Interworked WiMAX-3G Cellular Data Networks: An Architecture for Mobility Management and Performance Evaluation," IEEE Transactions on Wireless Communications, vol. 8, no. 4, pp. 1847-1853, Apr. 2009.

[3] S. Lee, K. Sriram, K. Kim, Y. H. Kim and N. Golmie, "Vertical Handoff Decision Algorithms for Providing Optimized Performance in Heterogeneous Wireless Networks," IEEE Transactions on Vehicular Technology, vol. 58, no. 2, pp. 865-881, Feb. 2009.

[4] Kaveh Shafiee, Alireza Attar and Victor C. M. Leung, "Optimal Distributed Vertical Handoff Strategies in Vehicular Heterogeneous Networks," IEEE Journal on Selected Areas in Communications, vol. 29, no. 3, pp. 534-544, Mar. 2011.

[5] Arslan Munir and Ann Gordon-Ross, "SIP-Based IMS Signaling Analysis for WiMAX-3G Interworking Architectures," IEEE Transactions on Mobile Computing, vol. 9, no. 5, pp. 733-750, May 2010.

[6] E. Stevens-Navarro, V. Shah-Mansouri and Vincent W. S. Wong, "Handoff Management and Admission Control Using Virtual Partitioning with Pre-emption in 3G Cellular/802.16e Interworking," IEEE Transactions on Vehicular Technology, vol. 59, no. 1, pp. 431-445, Jan. 2010.

[7] Zong-Hua Liu and Jyh-Cheng Chen "Design and Analysis of the Gateway Relocation and Admission Control Algorithm in Mobile WiMAX Networks," IEEE Transactions on Mobile Computing, vol. 11, no. 1, pp 5-18, Jan. 2012.

[8] P. Bellavista, A. Corradi and L. Foschini, "IMS-Compliant Management of Vertical Handoffs for Mobile Multimedia Session Continuity," IEEE Communications Magazine, vol. 48, no. 4, pp 114-121, Apr. 2010

[9] L. Nithyanandan and I. Parthiban "Seamless Vertical Handoff in Heterogeneous Networks Using IMS Technology," Proceedings of IEEE International Conference on Communication \& Signal Processing - 2012, Melmaruvathur, pp. 32-35, April 2012. 
International Journal of Wireless \& Mobile Networks (IJWMN) Vol. 4, No. 4, August 2012

\section{Authors Biography}

L. Nithyanandan received Bachelor of Engineering from University of Madras in 1992, Master of Technology in 1999 and Ph.D. degree in 2006 from Pondicherry University. $\mathrm{He}$ is working as an Associate Professor of Electronics and Communication Engineering, Pondicherry Engineering College, Puducherry, India. $\mathrm{He}$ is a gold medalist in PG and has been awarded with chief minister medal for his outstanding performance in PG. He has more than 22 publications in National / International conferences and Journals. His areas of interest include Sensor Networks, Telemedicine, Spread Spectrum Techniques and Wireless Communication.

I. Parthiban received his Bachelor of Technology and Master of Technology degrees in Electronics and Communication Engineering from Pondicherry University in 2010 and 2012 respectively. This journal was his maiden journal that has been published. His areas of interest include Embedded Systems, Networking and Wireless communication.
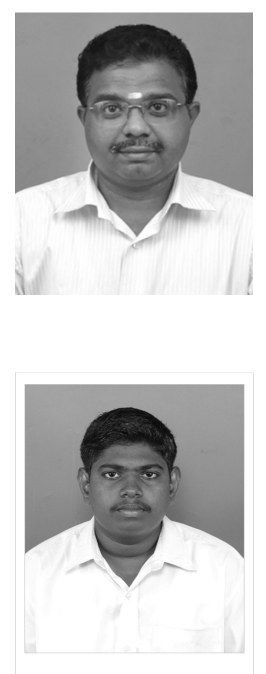